\title{
Forensic chemical profiling of flavouring additives in seized mu'assel (shisha) by gas chromatography-mass spectrometry (GC-MS)
}

Deepak Middha and Archna Negi ${ }^{*}$

\begin{abstract}
Background: Water-pipe tobacco smoking obsession has again spread worldwide. Especially, the younger generation is besotted with mu'assel (shisha) and avidly use these for smoking. Numbers of additives are being added in commercial brands of mu'assel (shisha) to impart diverse taste with amusing aroma. The name of only one of the masking flavours like strawberry, chocolate, vanilla etc. and not the chemical ingredients are printed on the packets of mu'assel. The manufactures remain silent about mentioning the chemical ingredients used for flavourings as they may not have scientific data about chemical compounds attributing to multiple flavours. There is also a shortfall in quality control owing to non-availability of technical procedure(s) to identify masking multiple flavouring additives. Many of these flavoured additives are either carcinogenic or potentially hazardous for human health. Ignoring health hazards, the avaricious manufacturers are intentionally adding multiple additives to make their products more addictive in order to increase their sales. The need of the hour is to unequivocally establish a technique for chemical profiling of the flavouring additives in mu'assel. In this paper, seven popular commercial brands of mu'assel were extracted, sonicated and analysed by GC-MS technique for detection of flavouring additives.

Results: Twenty-eight flavouring additives, i.e. camphor, linalool, benzyl ethanol, $\beta$-citronellol, menthol, vanillin, ethyl vanillin, eugenol, eucalptol, patchouli alcohol, nerol, rheosmin, musk ambrette, musk ketone, phenyl ethyl methyl ether, anethole, estragole, limonene, benzaldehyde, terpineol, phenyl ethyl butyrate, phenethyl isobutyrate, piperonal, methyl isobutyrate, methyl dihydro jasmonate, anisyl alcohol, trans-geraniol and sabinene along with nicotine were detected in varied proportions by GC-MS technique in seven seized popular commercial brands of mu'assel.
\end{abstract}

Conclusion: A study on chemical profiling of flavouring additives in commercial mu'assel has yet not been reported. Henceforth, this forensic attempt was aimed to secure public health by chemically profiling the flavouring additives of mu'assel. Many of the detected additives may cause severe health problems. Moreover, the smoker may suffer from neuroticism and psychoticism that may lead to a number of cases pertaining to physical assaults and sexual harassment.

Keywords: Forensic chemistry, Carcinogenic, Flavour, GC-MS, Nicotine, Smoking, Mu'assel, Hookah, Psychoticism, Public health

\footnotetext{
* Correspondence: archnaforensic@yahoo.com

Central Forensic Science Laboratory, Directorate of Forensic Science Services,

Ministry of Home Affairs, Govt. of India, Plot No. 2, Dakshin Marg, Sector 36

A, Chandigarh 160036, India
} 


\section{Background}

Tobacco is one of the most widely abused substances in the world and is found to be highly addictive for its major alkaloid stimulant-nicotine (Ralapati and $\mathrm{Lu}$ 1998). The tobacco plant is a part of the genus Nicotiana and of the Solanaceae (nightshade) family. While more than 70 species of tobacco are known, the chief commercial crop is Nicotiana Tabacum (Fig. 1).

Dried cured tobacco leaves are consumed by various ways such as in cigarettes, cigars, mu'assel, flavoured tobacco, snuff, chewing tobacco, dipping tobacco and snus. Among these, the most popular way to consume tobacco is mu'assel through a hookah or water pipe (Fig. 2) in which the vapour or smoke of mu'assel is passed through a water basin before inhalation (Wikipedia 2015). Mu'assel or shisha is becoming increasingly popular due to its flavoured tobacco smoke. It is a syrupy tobacco mix with molasses and vegetable glycerol as moisturiser. Some specific flavours are also added to it. Typical flavours of mu'assel include strawberry, chocolate, vanilla, apple, grape, guava, lemon and mint, as well as many other fruit-based mixtures. In this form, the user gets nicotine along with multiflavour smoke.

Additives are used primarily for flavours and smoothing the smoker's experience (Rabinoff et al. 2007). Ninety-five percent of chemicals used in fragrances are synthetic compounds derived from petroleum. Smoking them can cause cancer, birth defects, CNS disorders and allergic reactions, e.g. limonene-carcinogenic, benzyl alcohol-CNS depressants, camphor-CNS stimulant and linalool-CNS depressants (EPA 2015).

Some of these chemicals are also found addictive, e.g. menthol increases nicotine receptor density (Callier V 2014). Henceforth, the Tobacco Products Directive (2001/37/EC) was provided for EU member states to regulate tobacco products so as to protect public health. This directive included the prohibition of ingredients which increase the addictiveness of tobacco products (Europa 2015). Owing to the short falls in quality control norms and non-availability of a reliable facility to identify flavouring additives, avaricious manufacturers are intentionally adding additives without considering the adverse effect of these chemicals. Moreover, these manufacturers are only printing the name of the masking flavour and not the details of the other additives on the cover of the commercial mu'assel. Due to these unfair practices, smokers remain unaware about the health hazard of flavouring additives present in mu'assel. Some methods were developed for the detection of nicotine from tobacco leaves by GCMS (Hossain AM et al. 2013) and for detection of furanic compounds in the smoke of the water pipe (Schubert et al. 2011).

Study on chemical profiling of flavouring additives in mu'assel has not yet been reported. Henceforth, a

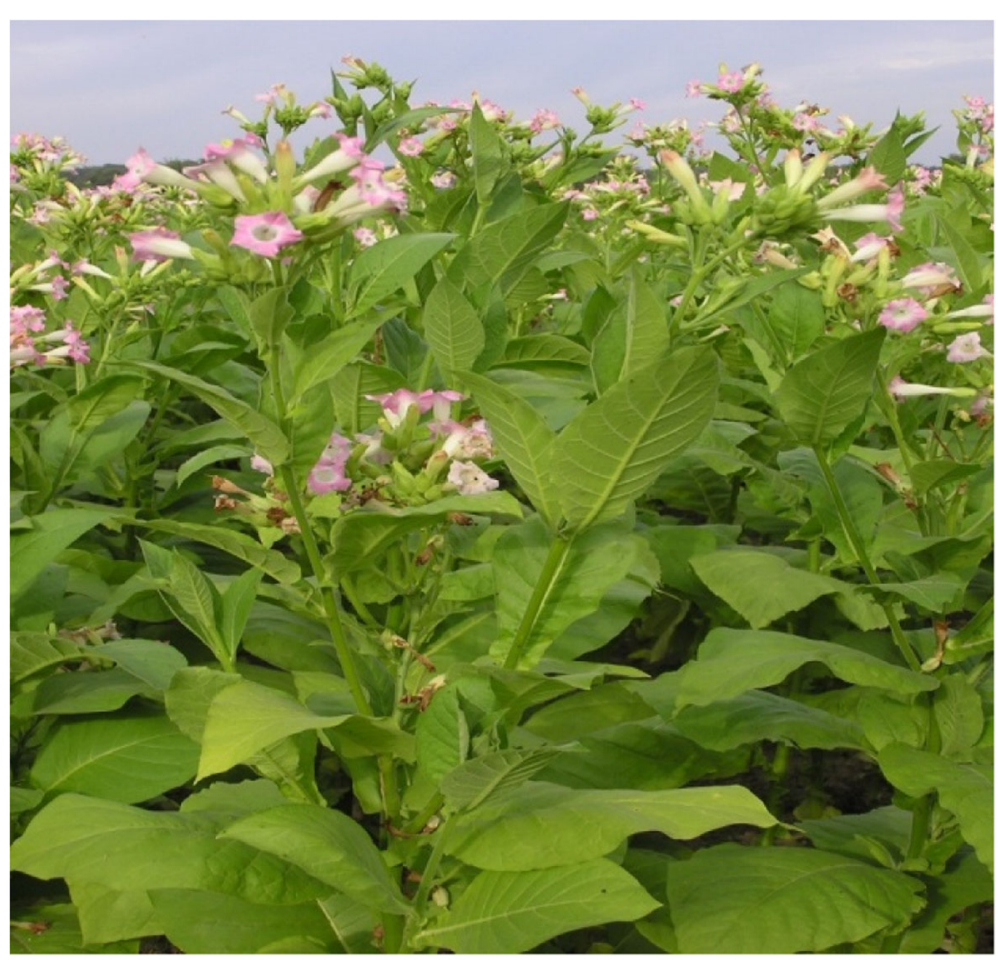

Fig. 1 Nicotiana Tabacum (Wikipedia 2015) 


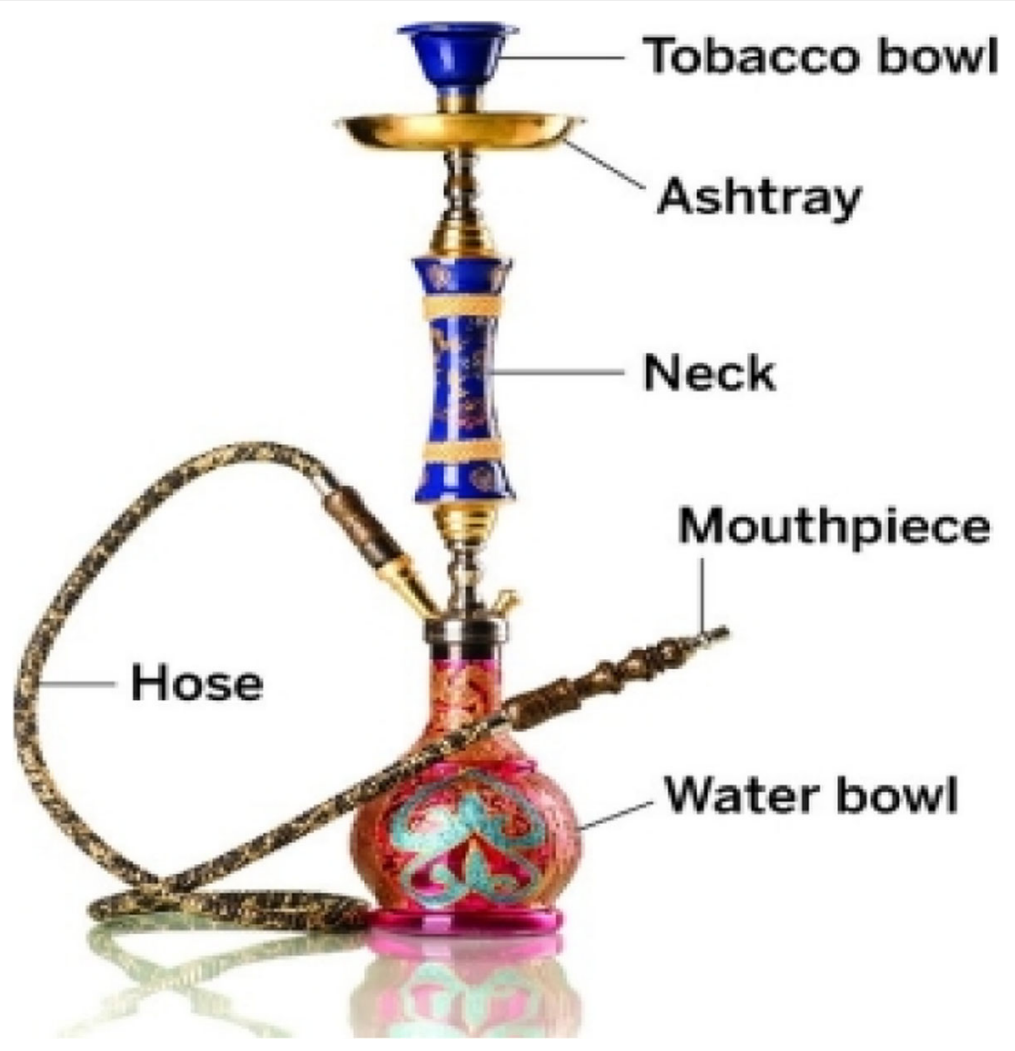

Fig. 2 Hookah (Wikipedia 2015)

forensic attempt is made to secure public health by chemically profiling flavouring additives in mu'assel. Forensic study was mainly targeted to give complete forensic chemical profiling of mu'assel so as to make smokers aware of the hidden health hazards of these products. In this study, representative samples from a large number of commercial varieties of mu'assel were subjected to forensic chemical analysis. These commercial varieties of mu'assel were seized during raids conducted in different hookah bars in Chandigarh by Chandigarh Police under COPTA Act, 2003, and were submitted at the Central Forensic Science Laboratory, Chandigarh, for the detection of nicotine. The samples of commercial varieties of mu'assel were ultrasonicated (Verma and Middha 2010) and analysed by using gas chromatography-mass spectrometry (GC-MS) technique. Twenty-eight flavouring additives apart from nicotine were identified by the mass spectral chemical profiling.

\section{Materials and method}

\section{Representative varieties and reagents}

The representative samples were taken from seven commercial varieties of mu'assel seized by Chandigarh police and were marked as R.V-1 to R.V-7.

\begin{tabular}{lll}
\hline Variety & Brand & Representative samples \\
1 & Aladdin "Lady Dragon" & R.V-1 \\
2 & Aladdin "Sweet 16" & R.V-2 \\
3 & Aladdin "Brain Freezer" & R.V-3 \\
4 & Aladdin "X on Beach" & R.V-4 \\
5 & Qehwa "Pan Salsa" & R.V-5 \\
6 & Mya"Bombay Blue Pan " & R.V-6 \\
7 & Mya "Pan Rasna" & R.V-7 \\
\hline
\end{tabular}

The packets of all the seized varieties of mu'assel were printed with only details of nicotine and tar, whereas the details of other multiple flavouring additives were not mentioned on any of the packets of mu'assel.

The solvent used for extraction was of LC grade (Merck, German).

\section{Equipment}

Ultrasonicator of PCi, Mumbai, India; Balance-AB104S of Mettler, Toledo, Switzerland; Thermo Finnigan Trace GC Ultra coupled with a Thermo DSQ Quadrupole MS and Thermo autosampler 3000 were used (directive 2001/37/EC). 
Ultrasonic extractions of mu'assel for flavouring additives Ten millilitres of chloroform was added to a 2-g representative sample of each variety in a round bottom flask and kept for $8 \mathrm{~h}$ in a refrigerator. These were then ultrasonicated for 1 followed by filtration through Whatman No.1 filter paper (GE Healthcare, UK). The filtrated samples were concentrated to $2 \mathrm{ml}$ and stored at $4{ }^{\circ} \mathrm{C}$.

\section{Instrumentation conditions}

A Thermo Finnigan Trace GC Ultra coupled with a Thermo DSQ Quadrupole MS and Thermo autosampler 3000 was used. The column was a 30-m BP-5 with 0.3$\mathrm{mm}$ I.D. and 0.5- $\mu \mathrm{m}$ film thickness. Helium was used as a carrier gas at a constant flow of $1.2 \mathrm{ml} / \mathrm{min}$. Splitless injection was used with a splitless time of $60 \mathrm{~s}$. The injector and interface line temperature were held at $250{ }^{\circ} \mathrm{C}$ and $330{ }^{\circ} \mathrm{C}$ respectively. Oven temperature was held at $90{ }^{\circ} \mathrm{C}$ for $1 \mathrm{~min}$ and increased to $310{ }^{\circ} \mathrm{C}$ at the rate of $20{ }^{\circ} \mathrm{CC} / \mathrm{min}$ and held at this temperature for $10 \mathrm{~min}$.

The MSD conditions: ionisation energy $70 \mathrm{eV}$, ion source temperature $200{ }^{\circ} \mathrm{C}$, mass range $41-410 \mathrm{amu}$, electron multiplier voltage (Auto tune $+200 \mathrm{~V}$ ).

Sample injection volume: $1 \mu \mathrm{l}$

\section{Compound identification}

Xcaliber 1.4 software was used for data acquisition and processing and results were screened using the library of National Institute of Standard and Technology.

\section{Results}

Various chemicals apart from nicotine were detected in R.V-1 to R.V-7 by GC-MS technique. Out of these, 28

Table 1 Detected flavouring additives in R.V-1

\begin{tabular}{llll}
\hline$R_{\mathrm{t}}$ & $\begin{array}{l}\text { Chemical } \\
\text { compound }\end{array}$ & $\begin{array}{l}\text { Molecular } \\
\text { weight } \\
(\mathrm{g} / \mathrm{mol})\end{array}$ & \\
\hline 5.54 & Limonene & 136.24 & $68,67,93,53,94,136$ \\
6.30 & Linalool & 154.25 & $71,93,55,121,136,139$ \\
6.51 & Benzene ethanol & 108.14 & $91,92,122,65,39,123$ \\
6.91 & camphor & 152.24 & $95,81,69,108,152,153$ \\
7.16 & Menthol & 156.27 & $71,81,95,55,123,138$ \\
7.65 & B-Citronellol & 156.27 & $41,69,81,82,123,138$ \\
7.93 & Nerol & 154.25 & $41,69,93,123,136,154$ \\
8.31 & Anethole & 148.21 & $148,147,133,77,51,149$ \\
8.96 & Nicotine & 162.2 & $84,45,133,162,55,119$ \\
8.98 & Eugenol & 164.20 & $164,149,77,55,103,165$ \\
9.40 & Vanillin & 152.15 & $151,152,81,123,53,153$ \\
9.89 & Ethyl Vanillin & 166.18 & $137,166,109,81,29,167$ \\
10.66 & Rheosmin & 164.20 & $107,164,43,121,94,165$ \\
11.75 & Patchouli alcohol & 222.36 & $83,138,98,222,81,55$ \\
\hline & & & \\
\hline
\end{tabular}

Table 2 Detected flavouring additives in R.V-2

\begin{tabular}{|c|c|c|c|}
\hline$\overline{R_{\mathrm{t}}}$ & $\begin{array}{l}\text { Chemical } \\
\text { compound }\end{array}$ & $\begin{array}{l}\text { Molecular } \\
\text { weight (g/mol) }\end{array}$ & Important fragments \\
\hline 5.58 & Eucalptol & 154.249 & $81,71,108,69,111,154$ \\
\hline 6.16 & $\begin{array}{l}\text { Phenyl ethyl methyl } \\
\text { ether (Kewda ether) }\end{array}$ & 136.19 & $45,91,136,104,65,137$ \\
\hline 6.53 & Benzeneethanol & 108.14 & $91,92,65,122,39,123$ \\
\hline 6.93 & Camphor & 152.24 & $95,81,69,108,41,152$ \\
\hline 7.16 & Menthol & 156.27 & $71,81,95,41,123,138$ \\
\hline 7.65 & $\beta$-Citronellol & 156.27 & $41,69,81,82,123,138$ \\
\hline 7.98 & Estragole & 148.20 & $148,147,77,121,91,51$ \\
\hline 8.33 & Anethole & 148.21 & $148,147,117,77,51,149$ \\
\hline 8.84 & Piperonal & 150.13 & $149,150,63,121,65,62$ \\
\hline 8.95 & Nicotine & 162.2 & $84,133,162,42,119,65$ \\
\hline 8.98 & Eugenol & 164.20 & $164,149,77,103,55,165$ \\
\hline 9.42 & Vanillin & 152.15 & $151,152,81,123,153,154$ \\
\hline 9.89 & Ethyl vanillin & 166.18 & $137,166,109,81,29,167$ \\
\hline 11.75 & Patchouli alcohol & 222.36 & $83,138,98,222,81,55$ \\
\hline 12.91 & Musk ambrette & 268.26 & $253,43,91,77,268,115$ \\
\hline
\end{tabular}

flavouring additives/ chemicals were found responsible for flavour in these representative varieties. These 28 flavouring chemicals are tabulated against their respective detected retention time $\left(R_{\mathrm{t})}\right.$ in Tables $1,2,3,4,5,6$ and 7 and their resulting total ion chromatogram are depicted in Figs. 3, 4, 5, 6, 7, 8 and 9 respectively. Combined chemical profiling of these 28 flavouring additives

Table 3 Detected flavoured additives in R.V-3

\begin{tabular}{llll}
\hline$R_{\mathrm{t}}$ & $\begin{array}{l}\text { Chemical } \\
\text { compound }\end{array}$ & $\begin{array}{l}\text { Molecular weight } \\
(\mathrm{g} / \mathrm{mol})\end{array}$ & Important fragments \\
\hline 5.54 & Limonene & 136.24 & $68,67,93,94,136,137$ \\
5.86 & Sabinene & 136.23 & $93,91,71,41,136,137$ \\
6.30 & Linalool & 154.25 & $71,93,43,121,136,139$ \\
6.51 & Benzene ethanol & 108.14 & $91,92,65,122,39,123$ \\
6.91 & Camphor & 152.24 & $95,81,108,41,152,55$ \\
7.16 & Menthol & 156.27 & $71,81,95,55,123,138$ \\
7.35 & Terpineol & 154.25 & $59,93,121,136,81,139$ \\
7.65 & B-Citronellol & 156.27 & $41,69,81,82,123,138$ \\
7.91 & Trans-gerniol & 154.25 & $69,41,68,93,123,139$ \\
8.95 & Nicotine & 162.2 & $84,133,162,42,119,65$ \\
8.98 & Eugenol & 164.20 & $164,149,77,103,55,39$ \\
9.40 & Vanillin & 152.15 & $151,152,81,123,53,153$ \\
9.72 & Phenyl ethyl & 192.25 & $104,105,71,91,106,161$ \\
& butyrate & & $137,138,166,109,81,167$ \\
9.91 & Ethyl vanillin & 166.18 & $107,43,164,77,121,165$ \\
10.66 & Rheosmin & 164.20 & $253,43,91,77,268,115$ \\
12.91 & Musk ambrette & 268.26 & $43,279,128,294,115,129$ \\
13.93 & Musk ketone & 294.30 &
\end{tabular}


Table 4 Detected flavouring additives in R.V-4

\begin{tabular}{|c|c|c|c|}
\hline$\overline{R_{\mathrm{t}}}$ & $\begin{array}{l}\text { Chemical } \\
\text { compound }\end{array}$ & $\begin{array}{l}\text { Molecular } \\
\text { weight ( } \mathrm{g} / \mathrm{mol})\end{array}$ & Important fragments \\
\hline 5.60 & Eucalptol & 154.249 & $43,81,71,108,111,154$ \\
\hline 5.81 & Methyl isobutyrate & 102.13 & $43,40,71,87,102,103$ \\
\hline 6.16 & $\begin{array}{l}\text { Phenyl ethyl } \\
\text { methyl ether }\end{array}$ & 136.19 & $91,136,104,65,137,138$ \\
\hline 6.30 & Linalool & 154.25 & $71,43,93,55,121,136$ \\
\hline 6.51 & Benzene ethanol & 108.14 & $91,92,65,122,39,123$ \\
\hline 6.93 & Camphor & 152.24 & $95,81,41,108,109,152$ \\
\hline 7.16 & Menthol & 156.27 & $71,81,95,55,67,123$ \\
\hline 7.65 & $\beta$-Citronellol & 156.27 & $41,69,81,82,123,138$ \\
\hline 7.93 & Nerol & 154.25 & $41,69,68,93,123,136$ \\
\hline 8.31 & Anethole & 148.21 & $148,147,117,77,105,51$ \\
\hline 8.96 & Nicotine & 162.2 & $84,133,162,92,82,163$ \\
\hline 8.98 & Eugenol & 164.20 & $164,149,77,103,55,165$ \\
\hline 9.40 & Vanillin & 152.15 & $151,152,81,123,53,153$ \\
\hline 9.74 & $\begin{array}{l}\text { Phenethyl } \\
\text { isobutyrate }\end{array}$ & 192.254 & $104,43,71,105,106,42$ \\
\hline 10.66 & Rheosmin & 164.20 & $107,43,164,77,121,65$ \\
\hline 11.45 & $\begin{array}{l}\text { Methyl dihydro } \\
\text { jasmonate }\end{array}$ & 226.32 & $83,156,153,82,55,96$ \\
\hline 11.73 & Patchouli alcohol & 222.36 & $41,43,83,98,138,222$ \\
\hline 12.89 & Musk ambrette & 268.2 & $253,268,91,77,115,145$ \\
\hline 13.93 & Musk ketone & 294.30 & $43,279,128,294,115,129$ \\
\hline
\end{tabular}

detected in R.V-1 to R.V-7 are summarised in Table 8 and the flavour of each detected chemical along with its chemical structure is summarised in Table 9.

\section{Discussion}

Twenty-eight flavouring additives were detected in seven representative varieties of mu'assel by GC-MS technique summarised in Tables 1, 2, 3, 4, 5, 6, ,7 and

Table 5 Detected flavouring additives in R.V-5

\begin{tabular}{llll}
\hline$R_{\mathrm{t}}$ & $\begin{array}{l}\text { Chemical } \\
\text { compound }\end{array}$ & $\begin{array}{l}\text { Molecular } \\
\text { weight } \\
(\mathrm{g} / \mathrm{mol})\end{array}$ & Important fragments \\
\hline $\mathbf{5 . 6 0}$ & Eucalptol & 154.249 & $81,71,108,111,154,69$ \\
6.16 & $\begin{array}{l}\text { Phenyl ethyl methyl } \\
\text { ether (Kewda ether) }\end{array}$ & 136.19 & $91,136,45,65,104,137$ \\
6.53 & Benzene ethanol & 108.14 & $91,92,65,122,64,123$ \\
7.16 & Menthol & $156.27 \mid$ & $71,81,95,123,138,67$ \\
7.65 & B-Citronellol & 156.27 & $41,69,81,82,123,138$ \\
7.93 & Trans-gerniol & 154.25 & $69,41,93,123,139,154$ \\
8.96 & Nicotine & 162.2 & $84,133,162,42,119,163$ \\
9.40 & Vanillin & 152.15 & $151,152,153,81,123,53$ \\
11.75 & Patchouli alcohol & 222.36 & $83,138,98,222,81,55$ \\
12.91 & Musk ambrette & 268.2 & $253,43,91,268,77,115$ \\
\hline
\end{tabular}

Table 6 Detected flavouring additives in R.V-6

\begin{tabular}{llll}
\hline$R_{\mathrm{t}}$ & $\begin{array}{l}\text { Chemical } \\
\text { compound }\end{array}$ & $\begin{array}{l}\text { Molecular } \\
\text { weight } \\
(\mathrm{g} / \mathrm{mol})\end{array}$ & \\
\hline 4.75 & Benzaldehyde & 106.12 & $105,106,77,78,107$ \\
5.60 & Eucalptol & 154.249 & $43,81,71,108,111,154$ \\
6.17 & Phenyl ethyl methyl ether & 136.19 & $91,136,45,65,104,103$ \\
6.30 & Linalool & 154.25 & $71,93,55,69,121,136$ \\
6.53 & Benzene ethanol & 108.14 & $91,92,122,65,39,93$ \\
6.93 & Camphor & 152.24 & $95,81,69,108,41,152$ \\
7.16 & Menthol & 156.27 & $71,81,95,82,123,138$ \\
7.65 & B-Citronellol & 156.27 & $41,69,81,82,123,138$ \\
7.93 & Trans-gernaniol & 154.25 & $69,41,68,93,123,139$ \\
8.28 & Anisyl alcohol & 138.17 & $138,137,109,77,94,39$ \\
8.96 & Nicotine & 162.2 & $84,133,162,42,119,163$ \\
8.98 & Eugenol & 164.20 & $164,149,77,103,131,55$ \\
9.40 & Vanillin & 152.15 & $151,152,81,109,53,50$ \\
9.89 & Ethyl vanillin & 166.18 & $137,166,138,109,81,167$ \\
10.66 & Rheosmin & 164.20 & $107,164,43,77,121,65$ \\
11.75 & Patchouli alcohol & 222.36 & $83,138,98,222,81,55$ \\
\hline
\end{tabular}

8 and TICs of R.V-1 to R.V-7 are depicted in Figs. 3, 4, $5,6,7,8$ and 9 respectively. Some of these detected chemicals are derived from plants origin, some are synthesised and some are both: These are categorised as under:

Table 7 Detected flavouring additives in R.V-7

\begin{tabular}{llll}
\hline$R_{\mathrm{t}}$ & $\begin{array}{l}\text { Chemical } \\
\text { compound }\end{array}$ & $\begin{array}{l}\text { Molecular } \\
\text { weight } \\
(\mathrm{g} / \mathrm{mol})\end{array}$ & \\
\hline 5.60 & Eucalptol & 154.249 & $43,81,108,71,111,154$ \\
6.16 & $\begin{array}{l}\text { Phenylethylmethylether } \\
\text { (Kewda ether) }\end{array}$ & 136.19 & $45,91,136,104,65,137$ \\
6.30 & Linalool & 154.25 & $71,93,55,68,121,136$ \\
6.53 & Benzene ethanol & 108.14 & $91,92,122,65,39,123$ \\
6.93 & Camphor & 152.24 & $95,81,69,108,41,152$ \\
7.17 & Menthol & 156.27 & $71,81,95,55,123,138$ \\
7.65 & B-Citronellol & 156.27 & $41,69,81,82,123,138$ \\
7.91 & Trans-geraniol & 154.25 & $69,41,93,123,139,154$ \\
8.31 & Estragole & 148.20 & $148,147,77,121,91,51$ \\
8.95 & Nicotine & 162.2 & $84,133,162,82,92,163$ \\
8.98 & Eugenol & 164.20 & $164,149,77,103,55,165$ \\
9.40 & Vanillin & 152.15 & $151,152,81,123,53,153$ \\
11.75 & Patchouli alcohol & 222.36 & $41,43,83,98,138,222$ \\
12.91 & Musk ambrette & 268.26 & $253,268,43,91,77,115$ \\
13.93 & Musk Ketone & 294.30 & $43,279,128,294,115,129$ \\
\hline & & &
\end{tabular}




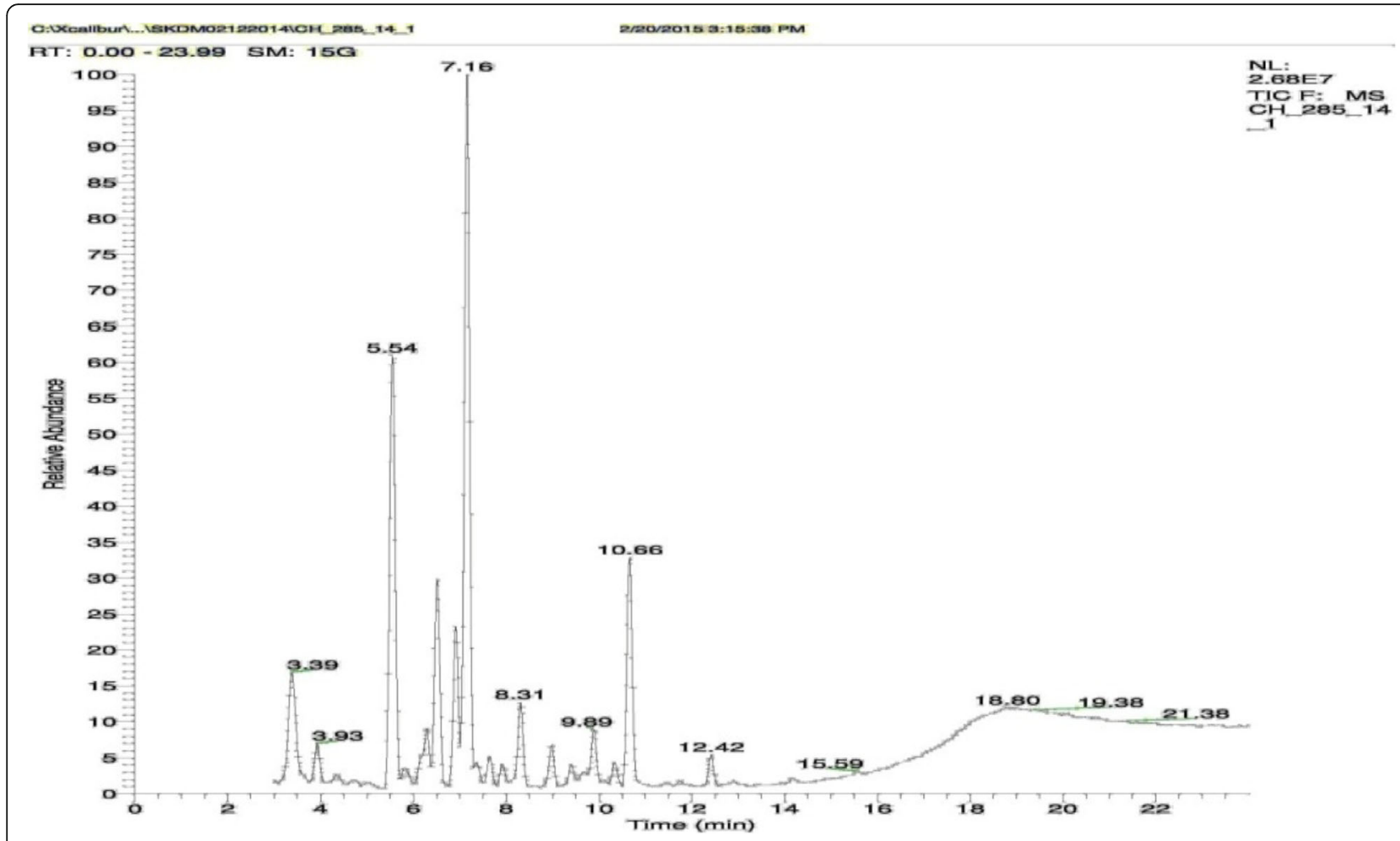

Fig. 3 Total ion chromatogram of R.V-1

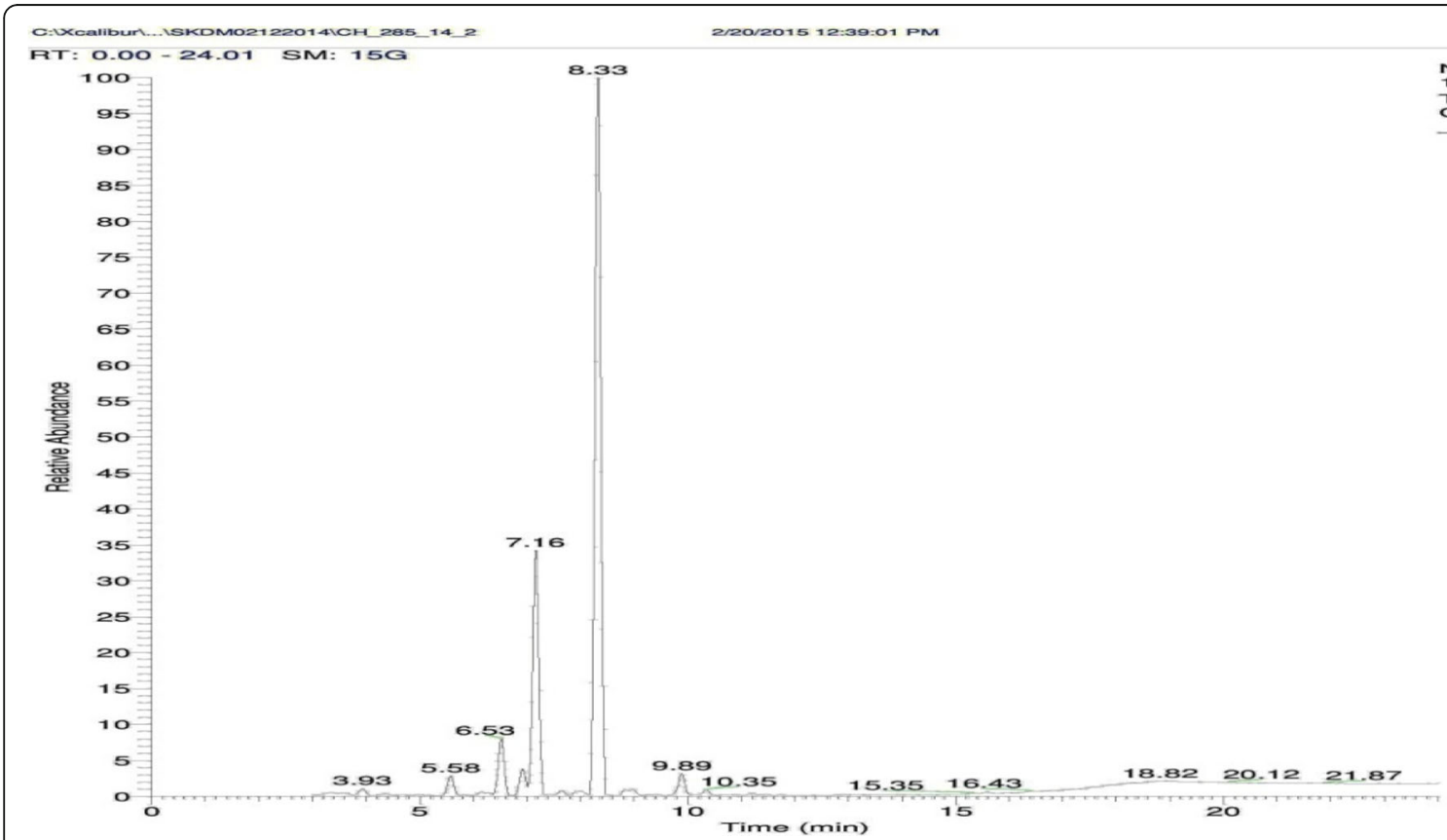

Fig. 4 Total ion chromatogram of R.V-2 


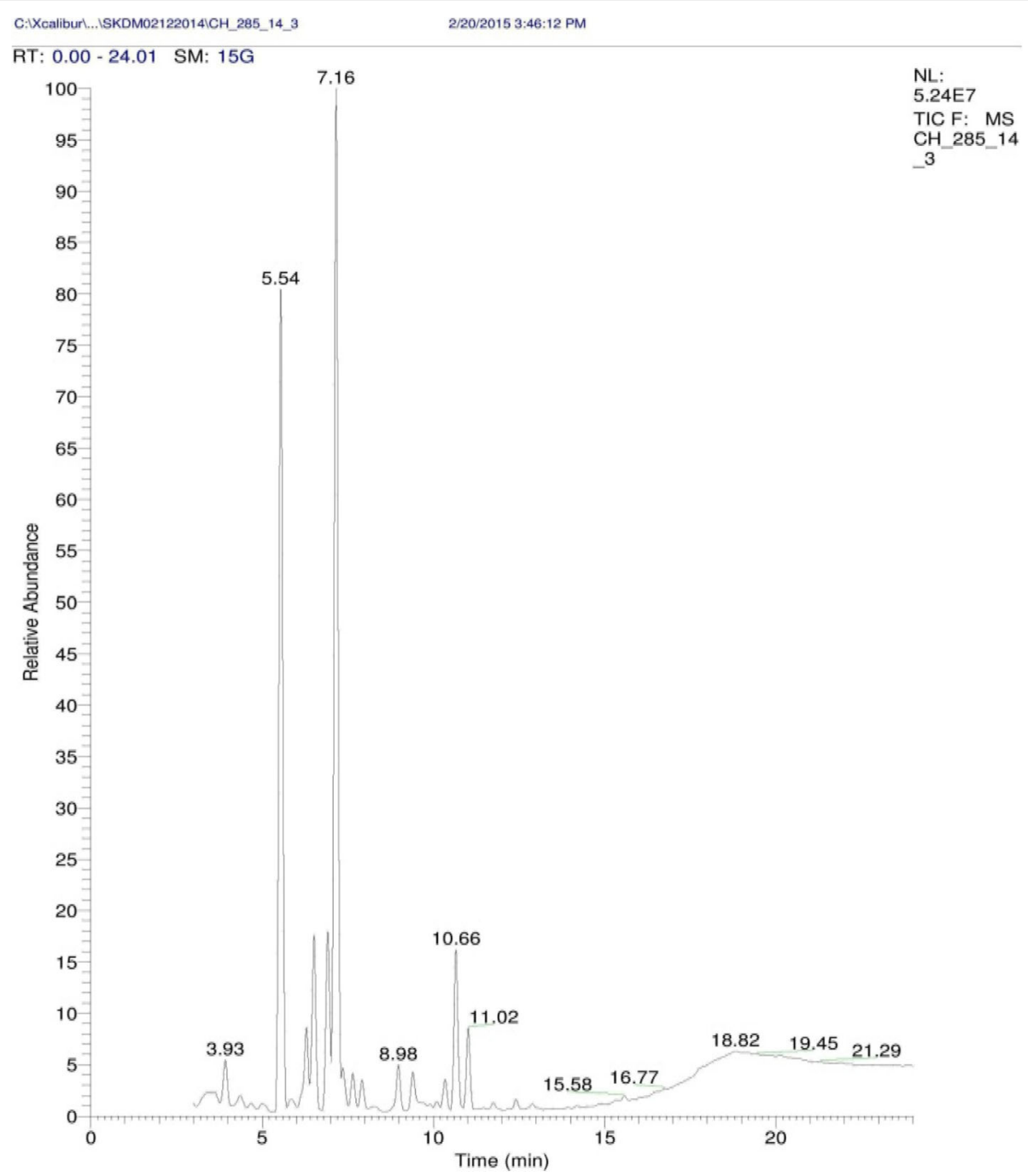

Fig. 5 Total ion chromatogram of R.V-3

1. Plant origin: camphor, linalool, benzyl alcohol, $\beta$ citronellol, menthol, vanillin, eugenol, patchouli alcohol, nerol, eucalptol, rheosmin, anethole, estragole, limonene, benzaldehyde, terpineol, trans-geraniol and sabinene

2. Synthetic: musk ambrette, musk ketone, ethyl vanillin, methyl isobutyrate, phenyl ethyl butyrate, phenethyl isobutyrate, phenyl ethyl methyl ether and methyl dihydro jasmonate

3. Both (plant origin as well as synthetic): piperonal and anisyl alcohol

These chemicals are added for imparting flavour into the mu'assel by the manufacturers. These detected chemicals owe specific properties for impartinga characteristic flavour. The flavour of each detected chemical is summarised in Table 9. Some of these chemicals impart similar flavours. The manufacturers inadvertently added on multiple chemicals imparting the same flavour in mu'assel, i.e for vanilla flavourvanillin and ethyl vanillin in R.V-1, R.V-2, R.V-3 and R.V-6; for spicy flavour-eugenol and eucalptol in R.V2, R.V-4, R.V-6 and R.V-7 and for musk aroma-musk ambrette and musk ketone in R.V-3, R.V-4 and R.V-7 and for aroma of mixture of citrus and floral- $\beta$ citronellol and methyl dihydro jasmonate in R.V-4.

TICs of seven representative varieties are depicted in Figs. 3, 4, 5, 6, 7, 8 and 9. Menthol was computed to have high proportion in R.V-1, R.V-3, R.V-5, R.V-6 and R.V-7, whereas anethole was in high proportion in R.V2 and R.V-4. Patchouli alcohol, benzene ethanol and camphor were detected in R.V-1, R.V-4, R.V-6 and R.V7; eucalptol in R.V-2 and R.V-4 to R.V-7; linalool in R.V-1, R.V-3, R.V-4, R.V-6 and R.V-7 and musk 


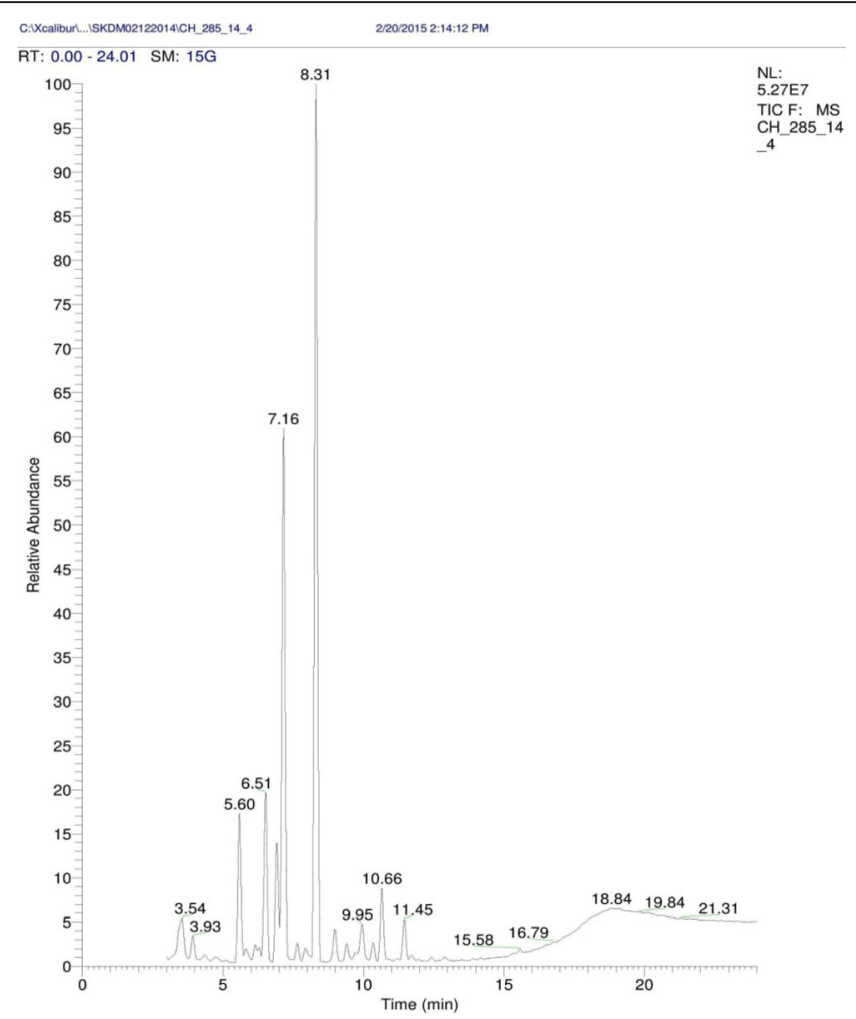

Fig. 6 Total ion chromatogram of R.V-4

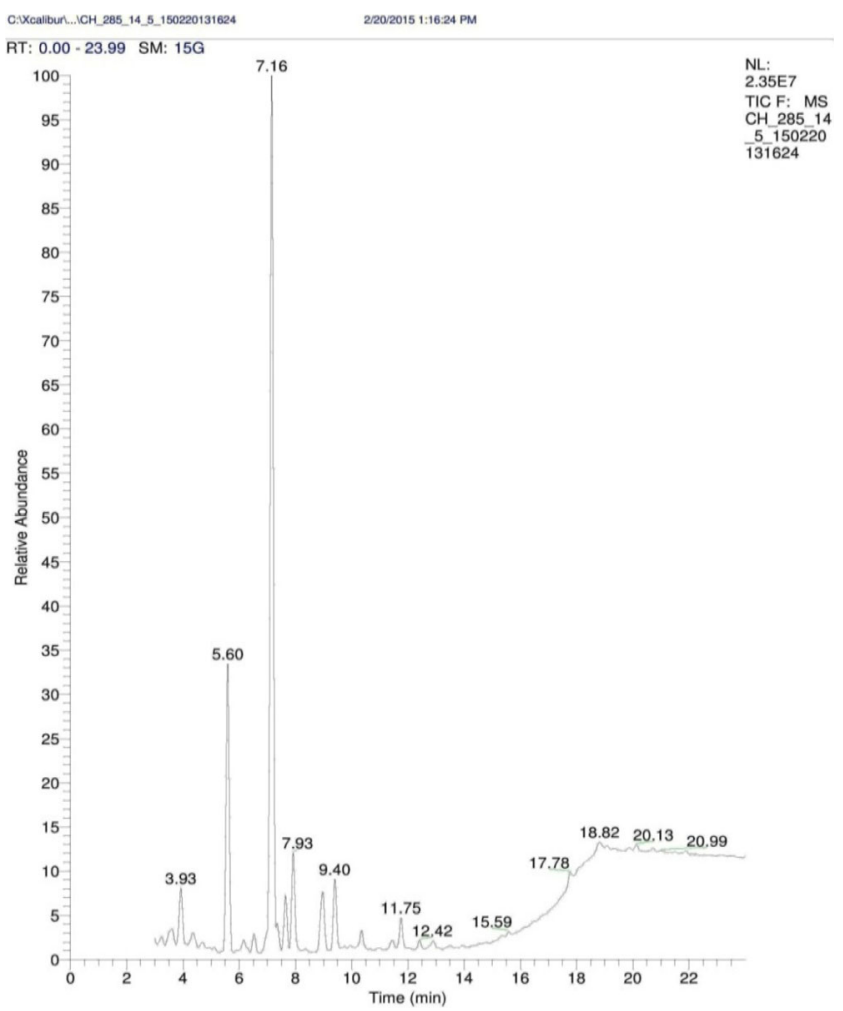

Fig. 7 Total ion chromatogram of R.V-5 


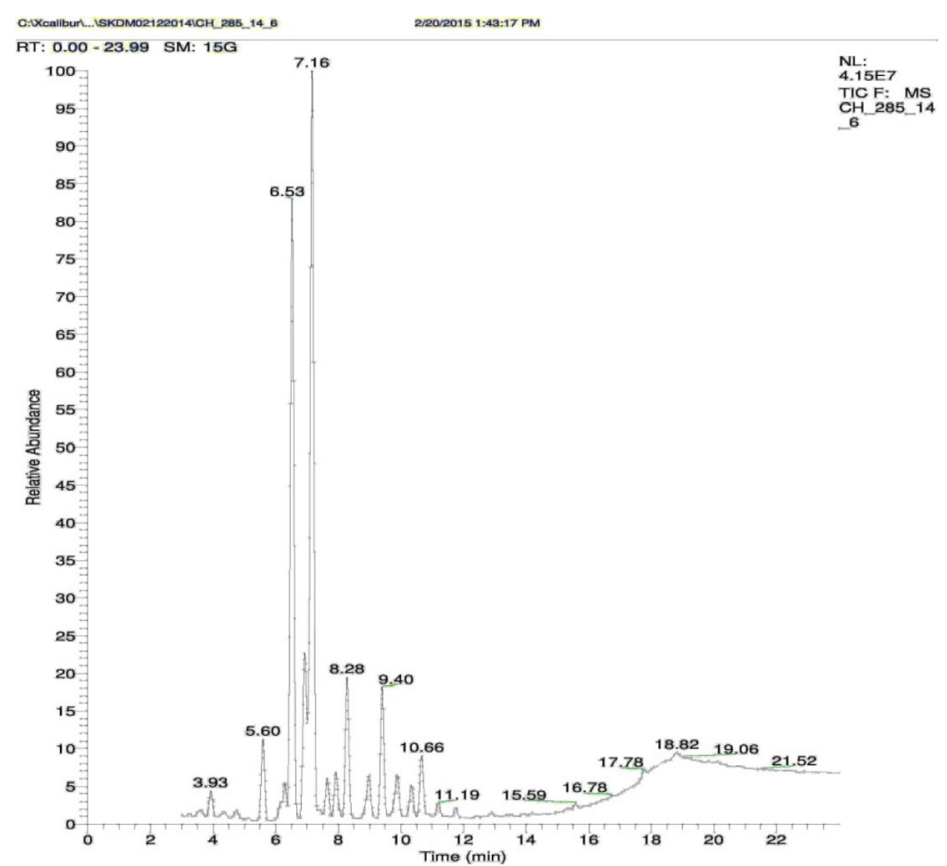

Fig. 8 Total ion chromatogram of R.V-6

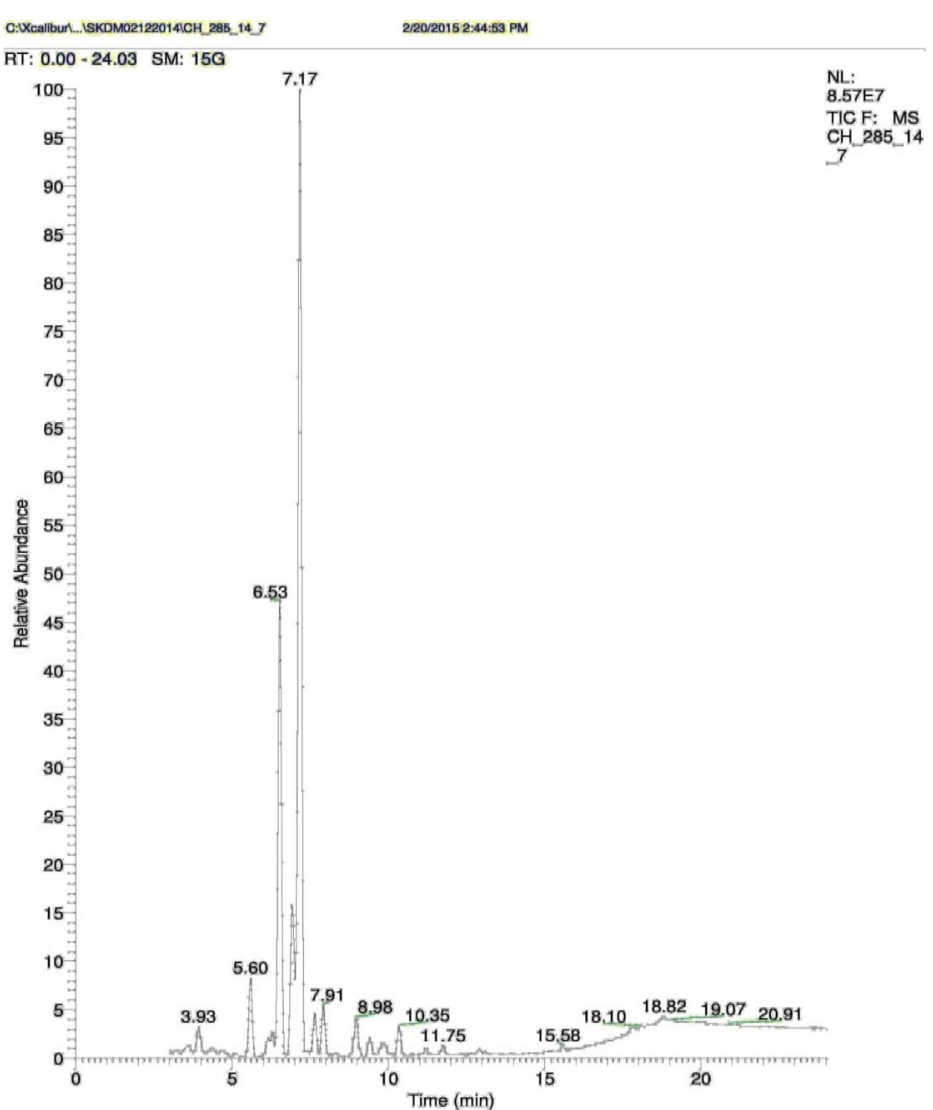

Fig. 9 Total ion chromatogram of R.V-7 


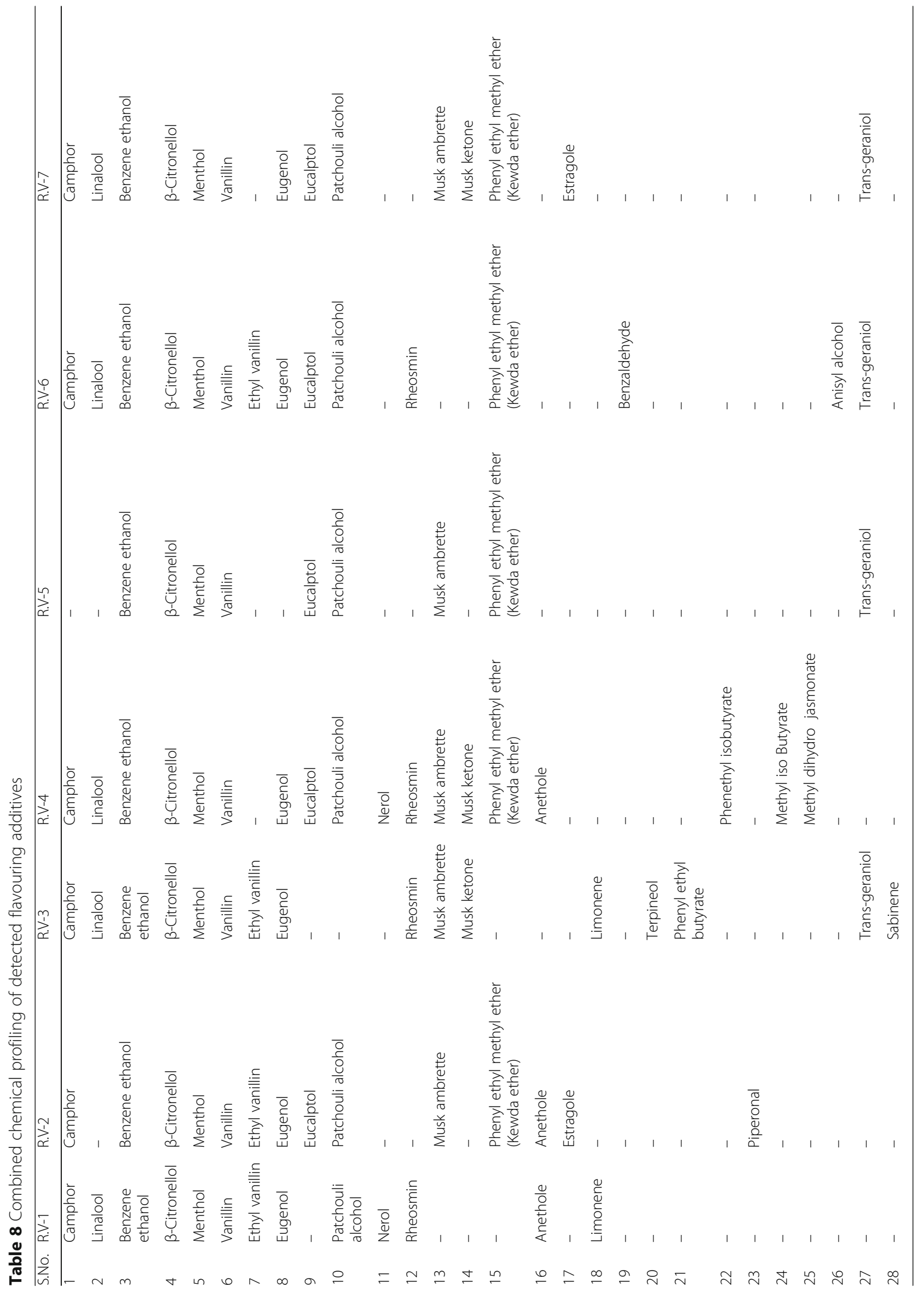


Table 9 Chemicals detected in R.V-1 to R.V-7 imparting the flavour

\begin{tabular}{|c|c|c|c|}
\hline S.No. & \begin{tabular}{|l|} 
Chemical compound \\
\end{tabular} & Structure & Flavour (aroma \& taste) \\
\hline 1. & $\begin{array}{l}\text { Camphor } \\
\text { Formula: } \mathrm{C}_{10} \mathrm{H}_{16} \mathrm{O} \\
\text { MW: } 152.24 \mathrm{~g} / \mathrm{mol}\end{array}$ & & $\begin{array}{l}\text { Strong penetrating fragrant } \\
\text { odour a bitter pungent taste }\end{array}$ \\
\hline 2. & $\begin{array}{l}\text { Linalool } \\
\text { Formula: } \mathrm{C}_{10} \mathrm{H}_{18} \mathrm{O} \\
\text { MW: } 154.25 \mathrm{~g} / \mathrm{mol}\end{array}$ & & $\begin{array}{l}\text { Pleasant scent (floral, with a } \\
\text { touch of spiciness) }\end{array}$ \\
\hline 3. & $\begin{array}{l}\text { Benzyl alcohol } \\
\text { Formula: } \mathrm{C}_{7} \mathrm{H}_{8} 0 \\
\text { MW:108.14g/mol }\end{array}$ & & Mild pleasant aromatic odour \\
\hline 4. & $\begin{array}{l}\beta \text {-Citronellol } \\
\text { Formula: } \mathrm{C}_{10} \mathrm{H}_{20} \mathrm{O} \\
\mathrm{MW}: 156.27 \mathrm{~g} / \mathrm{mol}\end{array}$ & & Flora, citrus-rosy, fresh, fruity \\
\hline 5. & $\begin{array}{l}\text { Menthol } \\
\text { Formula: } \mathrm{C}_{10} \mathrm{H}_{20} \mathrm{O} \\
\text { MW: } 156.27 \mathrm{~g} / \mathrm{mol}\end{array}$ & & Cool mint flavour \\
\hline 6. & $\begin{array}{l}\text { Vanillin } \\
\text { Formula: } \mathrm{C}_{8} \mathrm{H}_{8} \mathrm{O}_{3} \\
\text { MW: } 152.15 \mathrm{~g} / \mathrm{mol}\end{array}$ & & Vanilla taste and aroma \\
\hline 7. & $\begin{array}{l}\text { Ethyl vanillin } \\
\text { Formula: } \mathrm{C}_{9} \mathrm{H}_{10} \mathrm{O}_{3} \\
\text { MW: } 166.18 \mathrm{~g} / \mathrm{mol}\end{array}$ & & Vanilla taste and aroma \\
\hline 8. & $\begin{array}{l}\text { Eugenol } \\
\text { Formula: } \mathrm{C}_{10} \mathrm{H}_{12} \mathrm{O}_{2} \\
\text { MW: } 164.20 \mathrm{~g} / \mathrm{mol}\end{array}$ & & Spicy flavour \\
\hline 9. & $\begin{array}{l}\text { Eucalptol } \\
\text { (1,8-cineol, } 1,8 \text {-cineole) } \\
\text { Formula: } \mathrm{C}_{10}, \mathrm{H}_{18} \mathrm{O} \\
\text { MW: } 154.249 \mathrm{~g} / \mathrm{mol}\end{array}$ & & Pleasant spicy aroma and taste \\
\hline 10. & $\begin{array}{l}\text { Patchouli alcohol } \\
\text { Formula: } \mathrm{C}_{15} \mathrm{H}_{26} \mathrm{O} \\
\text { MW: } 222.36\end{array}$ & & Patchouli scent \\
\hline 11. & $\begin{array}{l}\text { Nerol } \\
\text { Formula: } \mathrm{C}_{10} \mathrm{H}_{18} \mathrm{O} \\
\text { MW: } 154.25 \mathrm{~g} / \mathrm{mol}\end{array}$ & & Sweet rose odour like geraniol \\
\hline 12. & $\begin{array}{l}\text { Rheosmin } \\
\text { Formula: } \mathrm{C}_{10} \mathrm{H}_{12} \mathrm{O}_{2} \\
\text { MW: } 164.20 \mathrm{~g} / \mathrm{mol}\end{array}$ & & Fruity odour of raspberry \\
\hline 13. & $\begin{array}{l}\text { Musk ambrette } \\
\text { Formula: } \mathrm{C}_{12} \mathrm{H}_{16} \mathrm{~N}_{2} \mathrm{O}_{5} \\
\text { MW:268.26584g/mol }\end{array}$ & & Musk type odour \\
\hline 14. & $\begin{array}{l}\text { Musk ketone } \\
\text { (white musk } \\
\text { Formula: } \mathrm{C}_{14} \mathrm{H}_{18} \mathrm{~N}_{2} \mathrm{O}_{5} \\
\text { MW:294.30312g/mol }\end{array}$ & & $\begin{array}{l}\text { Scent of deer musk or other } \\
\text { natural musk }\end{array}$ \\
\hline 15. & $\begin{array}{l}\text { Phenyl ethyl methyl ether } \\
\text { (kewda) } \\
\text { Formula: } \mathrm{C}_{9} \mathrm{H}_{12} 0 \\
\text { MW: } 136.19098 \mathrm{~g} / \mathrm{mol} \\
\end{array}$ & & $\begin{array}{l}\text { Green floral jasmine metallic } \\
\text { fresh rose note }\end{array}$ \\
\hline 16. & $\begin{array}{l}\text { Anethole } \\
\text { Formula: } \mathrm{C}_{10} \mathrm{H}_{12} \mathrm{O} \\
\text { MW: } 148.21 \mathrm{~g} / \mathrm{mol}\end{array}$ & & $\begin{array}{l}\text { Odour and flavour of anise } \\
\text { and fennel }\end{array}$ \\
\hline 17. & \begin{tabular}{|l} 
Estragole \\
Formula: $\mathrm{C}_{10} \mathrm{H}_{12} \mathrm{O}$ \\
$\mathrm{MW}: 148.20 \mathrm{~g} / \mathrm{mol}$
\end{tabular} & & $\begin{array}{l}\text { Anisic type odour and an } \\
\text { licorice type flavour }\end{array}$ \\
\hline 18. & $\begin{array}{l}\text { Limonene } \\
\text { Formula: } \mathrm{C}_{10} \mathrm{H}_{16} \\
\text { MW:136.24g/mol }\end{array}$ & & Strong smell of oranges \\
\hline 19. & $\begin{array}{l}\text { Benzaldehyde } \\
\text { Formula: } \mathrm{C}_{7} \mathrm{H}_{6} \mathrm{O} \\
\mathrm{MW}: 106.12 \mathrm{~g} / \mathrm{mol}\end{array}$ & & Almond like odour \\
\hline 20. & $\begin{array}{l}\text { Terpineol } \\
\text { Formula: } \mathrm{C}_{10} \mathrm{H}_{18} \mathrm{O} \\
\text { MW: } 154.25 \mathrm{~g} / \mathrm{mol}\end{array}$ & & Pleasant odour similar to lilac \\
\hline 21. & $\begin{array}{l}\text { Phenethyl butyrate } \\
\text { Formula: } \mathrm{C}_{12} \mathrm{H}_{16} \mathrm{O}_{2} \\
\text { MW: } 192.25 \mathrm{~g} / \mathrm{mol}\end{array}$ & & Floral odour \\
\hline 22. & $\begin{array}{l}\text { Phenethyl isobutyrate } \\
\text { Formula: } \mathrm{C}_{12} \mathrm{H}_{16} \mathrm{O}_{2} \\
\text { MW:192.254 } \mathrm{g} / \mathrm{mol}\end{array}$ & & $\begin{array}{l}\text { Sweet Fruity- rose honey floral } \\
\text { odour }\end{array}$ \\
\hline 23. & $\begin{array}{l}\text { Piperonal ( heliotropin) } \\
\text { Formula: } \mathrm{C}_{8} \mathrm{H}_{6} \mathrm{O}_{3} \\
\mathrm{MW}: 150.13 \mathrm{~g} / \mathrm{mol}\end{array}$ & & $\begin{array}{l}\text { Floral odour similar to that of } \\
\text { vanillin or cherry }\end{array}$ \\
\hline 24. & $\begin{array}{l}\text { Methyl iso butyrate } \\
\text { Formula: } \mathrm{C}_{5} \mathrm{H}_{10} \mathrm{O}_{2} \\
\text { MW:102.1317g/mol }\end{array}$ & & $\begin{array}{l}\text { Fruity odour of apple or } \\
\text { pinapple }\end{array}$ \\
\hline 25. & $\begin{array}{l}\text { Methyl dihydro jasmonate } \\
\text { or Hedione } \\
\text { Formula: } \mathrm{C}_{13} \mathrm{H}_{22} \mathrm{O}_{3} \\
\text { MW: } 226.32 \mathrm{~g} \cdot \mathrm{mol}^{-1}\end{array}$ & & $\begin{array}{l}\text { Mixture of Floral and citrus } \\
\text { odour }\end{array}$ \\
\hline 26. & $\begin{array}{l}\text { Anisyl alcohol } \\
\text { Formula: } \mathrm{C}_{8} \mathrm{H}_{10} \mathrm{O}_{2} \\
\text { MW: } 138.17 \mathrm{~g} \cdot \mathrm{mol}^{-1} \\
\end{array}$ & & Odour like hawthorn \\
\hline 27. & $\begin{array}{l}\text { Trans-Geraniol } \\
\text { Formula: } \mathrm{C}_{10} \mathrm{H}_{18} \mathrm{O} \\
\text { MW: } 154.25 \mathrm{~g} \cdot \mathrm{mol}-1\end{array}$ & & Rose like floral flavour \\
\hline 28. & \begin{tabular}{|l|} 
Sabinene \\
Formula: $\mathrm{C}_{10} \mathrm{H}_{16}$ \\
MW: $136.23 \mathrm{~g} / \mathrm{mol}$
\end{tabular} & & $\begin{array}{l}\text { Woody and Spiciness of black } \\
\text { pepper flavour }\end{array}$ \\
\hline
\end{tabular}


ambrette in R.V-2 to R.V-5 and R.V-7. $\beta$-citronellol, menthol, vanillin and benzene ethanol were detected in all seven representative varieties of mu'assel.

The collective flavour of these chemicals in seven representative varieties of mu'assel are summarised in Table 10.

For imparting higher flavour notes, the avaricious manufacturers add multiple chemicals un-proportionally to mu'assel without considering their hazardous consequences. Moreover, to increase the sales of their products, manufacturers have been adding multiple chemical compounds having alike flavour. Owing to these, the fusion of flavours of these multiple chemical compounds in mu'assel does not have any specific pleasant flavour due to interference of flavours of various chemicals.

\section{Conclusion}

Twenty-eight chemicals responsible for flavour were detected in seven representative varieties of mu'assel. The presence of these flavoured additives varies in each of the seven representative varieties. A difference in the flavoured additives causes a difference in the flavour of these samples. These flavoured additives not only add flavour to these mu'assel products but also make the person more addictive for its taste. Moreover, buyers are not aware of these chemicals and their harmful effects as the details of these flavoured chemicals are not printed on covers of any of these representative varieties. Therefore, the manufacturer should print the name of all the added chemicals on covers of mu'assel packets.

The users while using hookah inhale nicotine along with these chemicals. Some of the chemicals along with nicotine are accumulated inthe human body while some are converted to new compounds during the heating process and also get accumulated in the human body. These chemicals and their new converted chemicals are harmful to the body. Some of these chemicals have been approved as additives to food but these are not tested by burning. The aforementioned health hazards need utmost attention by the public authorities; on our part, we have indeed carried out an in-depth analysis on the burnt flavouring additives of Mu'assel vis-à-vis health issues. The findings are being critically reviewed before making in public.

\section{Forensic significance}

Production and consumption of flavoured mu'assel are based on various economic, social, cultural and government policies. Due to loopholes in the government policy, they are commercially available in plenty in the market/online purchase and especially in hookah bars. The consumption of hookah along with flavoured additives is a major issue related to health as these are so hazardous that their exposure to the human body can cause inflammation to monocytes and can cause severe health problems. Some of these are neurotoxic and some are even carcinogenic. Another aspect of the flavoured mu'assel is the increasing mortalities due to the disease caused by smoking hookah and by consumption of flavoured additives.

The hookah smokers tend to be more extroverted, tensed, impulsive, depressive, anxious and suffered from traits of neuroticism and psychoticism which results in a number of cases pertaining to physical assault and sexual harassment. To make the situation worse, there is no internationally accepted standard

Table 10 Collective flavour (taste and aroma) by flavouring additives in mu'assel varieties

\begin{tabular}{|c|c|c|}
\hline $\begin{array}{l}\text { Representative } \\
\text { sample }\end{array}$ & Collective taste & Collective aroma \\
\hline$\overline{R . V-1}$ & $\begin{array}{l}\text { Cool mint, vanilla, anise, fennel, citrus-rosy with } \\
\text { spiciness }\end{array}$ & $\begin{array}{l}\text { Strong penetrating odour of orange, anise, fennel, sweet rose, raspberry and } \\
\text { patchouli }\end{array}$ \\
\hline R.V-2 & $\begin{array}{l}\text { Cool mint, citrus-rosy, vanilla, licorice with } \\
\text { spiciness }\end{array}$ & $\begin{array}{l}\text { Strong penetrating odour of musk, green floral jasmine metallic fresh rose note, } \\
\text { anise and fennel anisic type odour. }\end{array}$ \\
\hline R.V-3 & $\begin{array}{l}\text { Cool mint, citrus-rosy, vanilla, woody with } \\
\text { spiciness of black pepper }\end{array}$ & Strong penetrating odour of musk, vanilla, raspberry, orange, lilac and rose \\
\hline R.V-4 & Cool mint, citrus-rosy, vanilla with spiciness & $\begin{array}{l}\text { Strong penetrating odour of patchouli, rose, spicy, raspberry, musk, green floral } \\
\text { jasmine metallic fresh rose note, anise, fennel, fruity odour of apple or pineapple, } \\
\text { sweet fruity-rose honey floral odour, mixture of floral and citrus }\end{array}$ \\
\hline R.V-5 & Cool mint, vanilla with spiciness & Citrus-rosy, patchouli, spicy, musk, green floral jasmine metallic fresh rose note \\
\hline R.V-6 & Cool mint, vanilla, spicy, rose-like floral & $\begin{array}{l}\text { Strong penetrating odour of citrus-rosy, patchouli, spicy, vanilla, fruity odour of } \\
\text { raspberry, green floral jasmine metallic fresh rose note and almond odour-like } \\
\text { hawthorn }\end{array}$ \\
\hline R.V-7 & $\begin{array}{l}\text { Floral, cool mint, vanilla, spicy, musk, licorice } \\
\text { type and rose-like floral }\end{array}$ & $\begin{array}{l}\text { Strong penetrating, floral with touch of spiciness, citrus-rosy, vanilla, spicy, } \\
\text { patchouli, musk, green floral jasmine metallic fresh rose note and anisic-type odour }\end{array}$ \\
\hline
\end{tabular}


method for analysing flavoured mu'assel in forensic cases along with any type of database.

This research publication does have a societical outreach as it makes the public at large aware about the hazardous effects of flavouring additives in a mu'assel and will aid the public authority to make a smoke-free world.

\section{Abbreviations}

CNS: Central nervous system; COPTA: Cigarette and Other Tobacco Product Act; EC: European community; EU: European Union; GC-MS: Gas chromatography-mass spectrometry; LC: Liquid chromatography; MSD: Mass selective detector; R.V: Representative variety; TIC: Total ion chromatogram; WHO: World Health Organization

\section{Acknowledgements}

The authors express their gratitude to the researchers engaged in restoring public health and safety and to the public authorities striving to make a smoke-free world

The authors are thankful to the Chief Forensic Scientist, DFSS, MHA, Govt. of India, and Director, CFSL, DFSS, MHA, Govt. of India, Chandigarh, for constant scientific support and encouragements.

\section{Authors' contributions}

Dr. DM has planned, reviewed, guided the research experimentations, interpretation and publication frame work. Mrs. AN has participated in methodology development, experimentations, interpretation and wrote the manuscript. Both authors have read and approved the final manuscript

\section{Authors' information}

Dr. Deepak Middha M.Sc., Ph.D. (F.S), LL.B. (Professional)

Working as Deputy Director \& Scientist-D in Chemistry division of Central Forensic Science Laboratory, Directorate of Forensic Science Services, Ministry of Home Affairs, Govt. of India, Chandigarh, India. He has brilliantly served for more than 29 years by making finest real-time applications of multi-scientific disciplines for the purpose of Criminal Justice. He has about three decades of professional experience and expertise in forensic chemical examinations and reporting on evidential clues pertaining to multi-type crimes involving bombing investigations, explosives, narcotic drugs, psychotropic substances, liquor, wildlife, bribery, homicide, arson, theft, burglary etc. He has successfully deposed hundreds of expert evidences in different Hon'ble Court of Law. He had the specialisation on the different mass-spectrometric techniques. He has contributed his services in CWs-MS interpretation (United Nations) multiple assignments. He has also made international contributions in the research and development sector.

Mrs. Archna Negi M.Sc(F.S), PGDCA

Serving in the same organisation as Senior Scientific Assistant in Chemistry Division. She is persuasive and contributing researcher and having more than eight years of experience in forensic chemical examinations on evidential clues pertaining to multi-type crimes involving, narcotic drugs, psychotropic substances, bribery, liquor, arson etc. She has also experience in examination of questioned documents pertaining to various problems in handwriting, typewriting, decipherment of altered and obliterated documents, printed matters including security documents such as currency notes and passport.

\section{Funding}

The authors received no specific funding for this work

\section{Availability of data and materials}

Related literature is available to authors

Ethics approval and consent to participate

Not applicable.

Consent for publication

Not applicable.

\section{Competing interests}

The authors declare that they have no competing interests.

Received: 10 April 2019 Accepted: 26 June 2019

Published online: 13 July 2019

References

Callier V (2014) Menthol increases nicotine addiction by tweaking brain. https:// www.newscientist.com/article/dn26668-menthol-increase-nicotine-addictionby-tweaking-brain. Accessed 10 Dec 2015

Directive 2001/37/EC of the European parliament and of the council (2001) http://www.tobaccocontrollaws.org/legislation/country/european-union/laws. Accessed 20 Dec 2015

Hookah. https://cen.acs.org/articles/93/i25/s-Hookah-Healthier-Smoking-Cigarette. html Accessed 10 Dec 2015

Hossain AM, Salehuddin SM (2013) Analytical determination of nicotine in tobacco leaves by gas chromatography-mass spectrometry. Arabian J Chem 3:275-278. https://doi.org/10.1016/j.arabjc.2010.10.006

Nicotiana. https://en.wikipedia.org/wiki/Nicotiana\#/media/File:Tabak_9290019.JPG. Accessed 13 Dec 2015

Rabinoff M, Caskey N, Rissling A, Park C (2007) Pharmacological \& chemical effects of cigarette additives. Am J Public health 97(11):1981-1991

Ralapati S, Lu GH (1998) Application of High-performance capillary electrophoresis to the quantitative analysis of nicotine and profiling of other alkaloids in ATF-regulated tobacco products. Electrophoresis 19(1):19-26

Schubert J, Bewersdorff J, Luch A, Schulz TG (2011) Waterpipe smoke: A considerable source of human exposure against furanic compounds. Analytica Chimica Acta 709:105-112

Twenty most Common Chemicals in Thirty-one Fragrance Products based on 1991 EPA Study. http://www.ourlittleplace.com/chemicals.html. Accessed 19 Dec 2015

Verma R.S, Middha D (2010) Chroma 71:117. https://doi.org/10.1365/s1033700913

\section{Publisher's Note}

Springer Nature remains neutral with regard to jurisdictional claims in published maps and institutional affiliations.

\section{Submit your manuscript to a SpringerOpen ${ }^{\circ}$ journal and benefit from:}

- Convenient online submission

- Rigorous peer review

- Open access: articles freely available online

High visibility within the field

- Retaining the copyright to your article

Submit your next manuscript at $>$ springeropen.com 